\title{
Transformaciones sociales y conocimiento en un contexto de crisis hídrica en la provincia de Córdoba, Argentina
}

\author{
Adrián Koberwein \\ Instituto de Ciencias Antropológicas, Universidad de Buenos Aires / \\ Consejo Nacional de Investigaciones Científicas y Técnicas \\ adriankoberwein@gmail.com
}

RESUMEN

En el presente artículo analizo el lugar que ocupa el conocimiento en la producción de nuevas relaciones sociales y configuraciones institucionales en el contexto de una crisis hídrica en las Sierras Chicas de Córdoba, Argentina. Basándome en un abordaje etnográfico, analizo la manera en que la información (tanto de origen cientifico como no científico) sobre ciertos hechos y fenómenos relativos al estado de situación de la crisis oficia como conocimiento en diferentes - pero complementarias-maneras, al ser, por un lado, apropiada y utilizada como base de las estrategias de visibilización de diferentes agrupaciones y organizaciones no gubernamentales, y por otro, fundamento de las intervenciones activas sobre la crisis por estas agrupaciones, creando así relaciones de cohesión, pero también de conflicto. Mostraré cómo estas relaciones se imbrican en un proceso de creación de nuevas configuraciones institucionales en el cual una campaña electoral, las preocupaciones y acciones concretas de los habitantes en torno al ambiente, y el conocimiento sobre los «procesos naturales», entre otras dinámicas, son considerados como parte constitutiva de un proceso más general de producción de la sociedad.

Palabras clave: conocimiento, crisis hídrica, transformaciones sociales, producción de la sociedad. 


\section{Social transformations and knowledge in the context of a water crisis in the province of Córdoba, Argentina}

\section{ABSTRACT}

This article analyses the role of knowledge in the production of new social relations and institutional configurations in the context of a water crisis in Sierras Chicas, Córdoba, Argentina. Based on an ethnographic approach; it analyses the way in which factual information of scientific and non-scientific origin, operates as knowledge in two different but complementary ways: on the one hand, it is appropriated and used as the basis of visibility strategies by different groups and non-governmental organizations; on the other hand, it works as the ground for active interventions on the water crisis, generating both cohesive and conflictual relations. The article shows how these relations become embedded in the process of creation of knew institutional arrangements, in which an electoral campaign, the concerns and actions of the inhabitants regarding environmental problems, and the knowledge about «natural processes», among other dynamics, are considered part of a more general process of production of society.

Keywords: knowledge, water crisis, social transformations, production of society. 


\section{INTRODUCCIÓN}

Desde hace casi una década, los municipios y comunas de las Sierras Chicas de Córdoba $^{1}$, Argentina, se ven afectados por una crisis hídrica y socioambiental ${ }^{2}$ cuya magnitud y persistencia en el tiempo es hoy una de las principales preocupaciones de una gran diversidad de actores. Entre ellos, cooperativas de servicios públicos a cargo de la distribución de agua potable, organizaciones no gubernamentales, grupos ambientalistas, agrupaciones vecinales, así como funcionarios y políticos que trabajan en las agencias del Estado local, provincial y nacional. Durante largo tiempo centradas en la escasez de agua, las preocupaciones de esta diversidad de actores en torno a la crisis hídrica hoy se focalizan en la sobreabundancia. Si entre los años 2007 y 2014 el problema central de la crisis hídrica fue que el agua no alcanzaba, desde febrero de 2015, la preocupación consiste en evitar que el agua produzca, nuevamente, pérdidas materiales y víctimas fatales, ante una posible reiteración de las inundaciones provocadas por las crecidas en los ríos y arroyos serranos.

El presente artículo, focalizado en la época de escasez, se enmarca en una investigación realizada desde la antropología social cuyo objeto es analizar las formas en que los diferentes actores mencionados se relacionan entre sí en el contexto de los procesos de definición de metas públicas de planificación y acción concreta en torno a la crisis. La zona de investigación abarca una franja del faldeo oriental de las sierras que forma parte de un corredor, tal como suelen referirse a dicha zona los medios de comunicación, cuyo componente urbano

1 Córdoba es una de las veintitrés provincias que componen la República Argentina. Situada en la región centro, sus $165321 \mathrm{~km}^{2}$ hacen de ella la quinta provincia más extensa del país. La capital, de nombre homónimo, es la segunda ciudad con mayor cantidad de habitantes, luego de Buenos Aires, capital de la Argentina.

2 El uso de la cursiva en el texto remite a una apropiación de las palabras de los actores. Cuando estas se encuentran en párrafo aparte y en letra normal, se trata de una reproducción de sus palabras tal como se encuentran registradas. 
se extiende unos $45 \mathrm{~km}$ hacia el norte de la ciudad de Córdoba, capital de la provincia homónima. La parte de dicho corredor que incluye nuestra investigación se corresponde con las áreas de influencia de los municipios de Unquillo, Río Ceballos, Salsipuedes, Agua de Oro y La Granja, y de las comunas Cerro Azul y El Manzano (ver plano a continuación).

La provincia de Córdoba es una de las provincias con mayor profundidad histórica respecto de aquello que los ecólogos culturales llamarían la «manipulación ambiental activa» de los recursos hídricos; es decir, la intervención y modificación del medio ambiente a gran escala en virtud de la producción de un ambiente habitable para la población humana. Un aspecto de este fenómeno se expresa en la gran cantidad de embalses de agua que encontramos en la provincia y en el hecho de que el dique San Roque (ver plano), construido entre 1884 y 1889 con el propósito de abastecer de agua potable a la ciudad de Córdoba, es el primer gran embalse construido en la historia argentina.

\section{Plano de referencia, Sierras Chicas}

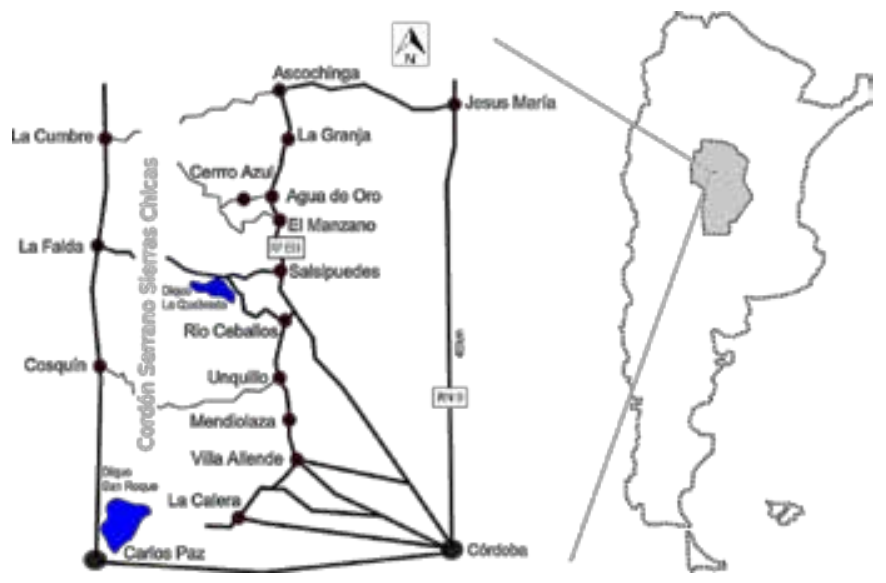

A diferencia de otras provincias con abundantes recursos hídricos, como Entre Ríos o Buenos Aires, en Córdoba la provisión de agua es un tema que alimenta la conversación pública en los niveles locales y regionales, ocupando un lugar considerable en las noticias de los medios de comunicación. En Córdoba, una crisis hídrica moviliza la participación de amplios sectores de la población, y no solo de los responsables de la gestión o el manejo del recurso. Aquí nos ocuparemos de una manera particular en que se movilizó esta participación durante una época de escasez de agua en la zona mencionada. 
En cuanto a la metodología, la investigación está basada en la puesta en práctica de herramientas etnográficas, tales como observación participante, entrevistas abiertas no dirigidas y charlas informales generadas durante las instancias de observación participante. Estas herramientas fueron implementadas bajo una modalidad de etnografía conocida como «multilocal» o «multisituada» (Marcus, 2001), que implica la estrategia de seguir, como afirma Marcus, las conexiones y relaciones entre diversos espacios locales, obligando así a romper con los límites etnográficos tradicionales ${ }^{3}$.

En esta oportunidad reflexionaré en torno a la manera en que cierto tipo de información es valorada por los actores en términos de los conocimientos necesarios para intervenir activa y efectivamente sobre la crisis, y esto en dos sentidos diferentes, pero articulados: por un lado, atenderé al lugar que ocupa la información - tanto de origen científico como aquella relativa a la experiencia cotidiana y que refiere al estado de situación de la crisis hídrica - en las estrategias de visibilización del accionar de las agrupaciones e instituciones; por otro, en el rol que dicha información cumple en la generación de instancias de acción e intervención activa sobre la crisis. Mi objetivo es dar cuenta de cómo cierta información, usada de determinada manera y en determinados contextos, se transforma en conocimiento en virtud de su lugar en la producción de nuevas relaciones sociales y configuraciones institucionales.

Quien ha enfatizado en forma más sistemática en la relación entre conocimiento y creación de relaciones sociales en la antropología fue Frederik Barth $(1995,2000)$. Barth compara dos modalidades de producción y circulación de

El holismo clásico de la etnografía queda, bajo esta modalidad, descentrado: no se trata de dar cuenta de la «totalidad» de la vida social en un espacio localizado, sino de rescatar la manera en que actores dispersos espacialmente, aunque unidos por un marco de referencia común — para el caso, la crisis hídrica-, actúan más allá de unos supuestos límites de una comunidad local de pertenencia. A lo largo de sucesivas campañas de trabajo de campo, con una temporalidad discontinua entre mediados de 2011 y 2015, he entrado en contacto con actores de las diferentes localidades de las Sierras Chicas, aquellos específicamente involucrados en forma directa en la política de los recursos hídricos: funcionarios y políticos, miembros de $\mathrm{ONG}$, agrupaciones de vecinos preocupados por la situación hídrica y ambiental, y cooperativas de servicios públicos que se encargan de la distribución del agua potable de red. Asimismo, he asistido una diversidad de eventos y reuniones en los cuales se trataron temas relativos al ambiente y al agua, y participé de manifestaciones organizadas por habitantes afectados por la crisis en diferentes localidades serranas. Cabe advertir que los diversos actores cuyos dichos se recuperan a lo largo del argumento, así como las diferentes situaciones que protagonizan, no necesariamente están vinculados en forma directa, pues forman parte de una dinámica regional común que excede sus ámbitos de acción e influencia inmediatos (para una ampliación al respecto, ver Koberwein, 2015). 
conocimiento. Una implica la creación de un conocimiento encapsulado, individualizado y transportable; allí reside su valor. En la otra modalidad, el valor del conocimiento aumenta cuando este es compartido, aunque por un reducido número de personas. Sin embargo, en el marco de la política de los recursos hídricos en Sierras Chicas, la información es valorada como conocimiento cuando es abierta, difundida y compartida entre el mayor número de personas posibles. Como veremos, el proceso mismo de buscar, compartir y hacer circular esta información implicó, para este contexto, un proceso de creación de relaciones sociales. Así, la búsqueda de información y su transformación en conocimiento se vuelve la «sustancia generadora» de las relaciones, tal como diría Barth (2000). Dicho en otras palabras: la información en sí misma, como una serie de datos factuales producidos - ya sea por la ciencia o a través de la experiencia y la acción de los protagonistas de los procesos que aquí abordamos- se transforma en conocimiento cuando moviliza e impulsa la producción de nuevas relaciones sociales.

A diferencia de otros animales sociales, dice Godelier, los seres humanos «no se contentan con vivir en sociedad, sino que producen la sociedad para vivir; en el transcurso de su existencia, inventan nuevas maneras de pensar y de actuar sobre ellos mismos así como sobre la naturaleza que los rodea» $(1989, \mathrm{p} .17)$.

Quisiera proponer a este respecto que, en contextos donde exista algún tipo de reconocimiento por los actores de la existencia de problemas sociales a resolver, y cuando dicho reconocimiento se transforme en acción para revertir aquellos problemas, estamos ante un campo analítico fértil para analizar estos procesos de producción de la sociedad. El supuesto que sostiene este enfoque es que aquello que llamamos «sociedad» es un sostenido proceso de producción (y reproducción), en el cual se ponen constantemente en juego «nuevas» formas de pensamiento, acción y organización social. El contexto social donde se inserta esta investigación presenta, justamente, este escenario.

Ahora bien, teniendo en cuenta el enfoque que estamos tomando aquí, deberíamos agregar que nuevas relaciones sociales solo pueden surgir si son pensables y consideradas como posibles en virtud de ciertos objetivos o necesidades construidas socialmente como tales (Godelier, 1989). En este sentido, el conocimiento al que hacemos referencia es, para el contexto de nuestro análisis, aquel que permite pensar, encauzar e impulsar la producción de nuevas relaciones. En este sentido, más que una «sustancia generadora», como diría Barth, el conocimiento oficia como una verdadera «fuerza productiva», en el sentido marxiano del término. Ambas formas de tratar el conocimiento no son, sin embargo, excluyentes. La propuesta de Barth nos permite considerar el carácter objetivo, maleable del 
conocimiento; por su parte, la idea marxista de «fuerza productiva» nos remite al carácter procesual del fenómeno. En este sentido, la «sustancia» se vuelve proceso, al ser reincorporada en nuevos contextos y nuevos usos.

Cabría dejar sentado que el concepto de producción que aquí utilizamos debe entenderse, en términos amplios y para nuestro contexto de análisis, como una actividad transformadora y socialmente organizada (Marx, 2005; Turner, 2008), en virtud del reconocimiento de una necesidad de cambio social. Terence Turner (2008), basándose en La ideología alemana de Marx y Engels (2005), afirma que allí la producción aparece definida como una praxis transformadora que consiste en cuatro aspectos principales: (i) la producción de medios de subsistencia; (ii) la producción de nuevas necesidades que dan origen a nuevas relaciones sociales; (iii) la producción de seres humanos, y (iv) la producción de diferentes relaciones de cooperación social. En esta línea, Turner argumenta que la concepción de Marx está anclada en la idea de que la producción material es, simultáneamente, un proceso de producción del sistema social ${ }^{4}$. Y es este segundo aspecto de la producción el que puede (y a su juicio debe) ser aplicado a contextos en los cuales la producción de bienes materiales o mercancías no es lo central para explicar los procesos sociales.

Con esto en mente, analizaré el lugar que ocupa el conocimiento en la creación de nuevas relaciones sociales (como un aspecto de la producción de la sociedad) en el marco de la política de los recursos hídricos en la zona mencionada; es decir, en un contexto en donde se despliegan las acciones de una serie de instituciones y agrupaciones sobre una crisis hídrica y socioambiental que genera una serie de problemas vitales que, a juicio de los actores, deben ser socialmente resueltos $\mathrm{o}$, al menos, enfrentados en un intento por resolverlos.

A los efectos del presente análisis, consideraré como definición operativa de conocimiento toda información que sea activamente buscada, producida o apropiada por los actores como fundamento y base de aquello que mencioné anteriormente. Es decir, (i) de las estrategias de visibilización, y (ii) de las intervenciones activas sobre la crisis. Como se trata de problemas ambientales, hay cierto tipo de información que se destaca: la información científica y técnica. Por lo tanto, este trabajo podría ser considerado como parte de una prolífica línea de

4 Si bien Turner se basa en La ideología alemana, se trata de un problema que Marx retoma en El capital de la siguiente manera: «El proceso capitalista de producción [...] no produce solamente mercancías, no produce solamente plusvalía, sino que produce y reproduce el mismo régimen del capital: de una parte al capitalista y de la otra al obrero asalariado» (Marx 2000[1867], p. 487. Tomo I. Resaltado en el original). 
estudios tales como los de Descola (1996, 2012), Escobar (1999), Ingold (2000), que analizan la manera en que la naturaleza es construida social y culturalmente; Leff (2000) quien enfatiza el lugar de los conocimientos locales de las cuestiones ambientales; Leite Lopes (2006), quien se ocupa de los procesos de ambientalización de los conflictos; Pinto Ribeiro (2012), quien se dedica a analizar los cambios de significados en la expresividad del ambientalismo; Hukkinen (2014), que se ocupa de la producción de conocimiento ambiental poniendo en tensión la teoría del conocimiento incorporado con la perspectiva del conocimiento como procesamiento de información, y muchos otros.

En la Argentina, cabe destacar los trabajos de Stagnaro (2015), que aborda, a partir del análisis de una serie de casos etnográficos, la manera en que el término cientifico «constituye una categoría cultural cargada de significados complejos y contradictorios susceptibles de ser utilizados e interpretados [...] en el contexto social de actuación» (2015, p. 319). Por su parte, Hidalgo y Stagnaro (2016) destacan las complejas relaciones entre la ciencia y políticas públicas, e Inés Vázquez (2016) analiza la manera en que la muestra «Tecnópolis» (2011-2015) buscó resaltar la valorización social de la ciencia y la tecnología. En cuanto a la relación entre conocimiento y las temáticas ambientales, destacan los trabajos de Ferrero (2006), Balbi (2007) y Macip (2015), quienes analizan la manera en que el «discurso ambientalista» es reapropiado en el ámbito de las actividades de pesca, agricultura familiar y ecoturismo, respectivamente. En cuanto a la producción de conocimiento científico sobre el ambiente, Blois (2016) indaga en torno al rol de los científicos en la producción del conocimiento sobre los efectos del uso de glifosato en la Argentina. Carabajal (2016), por su parte, aborda la manera en que se construye el conocimiento climático en el Servicio Meteorológico Nacional, y Martins (2008) nos revela el lugar que ocuparon las diferentes escuelas de politécnicos al momento de modernizar la gestión de cuencas en Francia.

Si bien el trabajo que aquí presento podría enmarcarse en una o varias de estas diferentes líneas, mi objeto no es analizar cómo se produce el conocimiento científico sobre el ambiente en sí sino el lugar que cierto tipo de información, valorada por su origen científico, ocupa en los procesos de producción social, entendida en los términos anteriormente expuestos. Sin embargo, la información de origen científico no es la única que se busca activamente. También se pone en juego otro tipo de información cuyo origen es más cercano a la vida cotidiana de los protagonistas: por ejemplo, respecto de qué medidas pretenden tomar los funcionarios y los políticos en el contexto de crisis, o del estado de situación de las redes de distribución de agua potable; la información sobre los niveles de los 
diques y embalses, e incluso sobre la forma como viven la crisis otras localidades de la zona y cómo la enfrentan. Mostraremos a continuación que el mismo proceso activo e intencional de búsqueda de información forma parte de un proceso más general de producción de relaciones sociales. Cuando la búsqueda y puesta en circulación de la información moviliza esta producción es cuando estamos hablando que se trata de conocimiento.

\section{LA CRISIS: EXPERIENCIA COTIDIANA, INFORMACIÓN Y CONOCIMIENTO}

En un sentido socioespacial, las Sierras Chicas están plagadas de tensiones y contradicciones. Su ambiente natural es, histórica y actualmente, la base de una amplia diversidad de actividades productivas que generan dinámicas muchas veces antagónicas entre sí. En las sierras conviven la actividad pecuaria, la actividad de las canteras, la industria turística, la actividad inmobiliaria y la construcción, por nombrar algunas. Algunos pueblos y localidades de las sierras están transformándose paulatinamente en «pueblos dormitorio»; es decir, lugares que reciben a familias y habitantes que deciden vivir fuera de la ciudad capital, pero que mantienen vínculos permanentes con ella en virtud de actividades laborales, educativas, etc. (Koberwein, 2015b). Las sierras son fuente de agua y otros recursos naturales que sostienen aquellas actividades productivas y dinámicas poblacionales que he mencionado y, en virtud del sostenido crecimiento poblacional de los últimos quince años, el tema de los recursos hídricos disponibles es objeto de un amplio debate público en toda la zona.

En general, los protagonistas de la política de los recursos hídricos en Sierras Chicas rastrean las primeras manifestaciones de la crisis hídrica en el año 2007 (con referencias siempre a una sequía anterior, en 2004), cuando el dique La Quebrada, único embalse de la zona de estudio, comenzó su carrera cíclica de bajante sin recuperarse sino hasta entrado el año 2014. Si bien el dique proveía por entonces solo a tres de las localidades ${ }^{5}$, el nivel de agua embalsada ofició siempre como una suerte de medida de la cantidad de agua disponible. La crisis hídrica se vive de maneras diversas en las distintas localidades, ya sea con mayor o menor intensidad según la época del año o según otras cuestiones que no vale la pena tratar aquí por razones de argumento. A pesar de ello, el nivel de agua del dique La Quebrada oficia como una suerte de parámetro objetivo de la intensidad

\footnotetext{
5 Mendiolaza, Unquillo y Río Ceballos.
} 
de la crisis hídrica en las sierras, sobre todo en los medios de comunicación, que publican diariamente las cifras de todos los embalses de la provincia, tomando a La Quebrada como la referencia para la vertiente oriental de las Sierras Chicas.

Se nos revela así un primer sentido concreto de la información: es un parámetro objetivo que sirve no solo para medir y ponderar un estado de situación presente — la falta de agua—, sino también para producir el reconocimiento de una necesidad de hacer algo al respecto, justificando e impulsando las primeras iniciativas en torno a una situación que comenzó a ser vivida y conceptualizada como una crisis. Una de estas iniciativas, encarada por los prestadores del servicio de agua potable, fue la puesta en práctica de un sistema de racionalización del consumo de agua a través de cortes programados en la distribución.

La cantidad de agua embalsada por el dique oficiaba, en este contexto, como una medida de la intensidad de la crisis. Las mediciones, como para todo embalse, se publican de la siguiente manera: tomando el vertedero del dique como el nivel cero, se calcula la distancia hasta el nivel del agua. En enero de 2010, el agua había retrocedido a un nivel récord de -13,02 m. En septiembre de 2011, el nivel indicaba $-11,81 \mathrm{~m}$. La publicación de las cifras al 18/06/2012 fue de $-6,08 \mathrm{~m}$.

Febrero de 2014 fue también un mes de récords: el 3 de febrero se alcanzó una nueva marca histórica de bajante del dique: $-14,80 \mathrm{~m}$. Las fuertes lluvias acaecidas durante ese mes hicieron que el dique se llenara, finalmente, luego de siete años de merma.

El carácter socialmente productivo de esta información se nos revela cuando consideramos que, en forma aislada, estas cifras no brindan una información técnica precisa, pues no expresan en sí mismas el volumen de agua faltante o, a la inversa, el agua retenida efectivamente por el dique. Para ello haría falta realizar un cálculo que incluyese su capacidad total de acumulación, la superficie cubierta por el agua en cada momento y el nivel de sedimentos en el lecho del lago, entre otros datos, con el fin de explicar técnicamente cuánta cantidad de agua faltante implica un metro lineal medido desde el vertedero. Sin embargo, no se necesita ser ingeniero hidráulico para comprender la información. En el lenguaje cotidiano, las cifras toman sentido en términos comparativos, y el resultado de su lectura no es «nos faltan tantos metros cúbicos de agua» sino: «estamos peor que el año pasado» o «nos faltan dos metros para alcanzar el récord». Así, la primera información que dio sentido a una crisis hídrica implicó cifras y niveles que conformaban un conocimiento sobre la escasez. El carácter productivo de esta información reside en que impulsó tanto la búsqueda de las razones como la elaboración de proyecciones a futuro sobre la situación de crisis, y sobre todo, 
sentó la necesidad de intervenir para paliar sus efectos negativos. Por lo tanto, nuevas formas de cooperación y organización social se volvieron socialmente necesarias.

La información de que el agua no alcanzaba para todos, valorada como conocimiento de un estado de situación presente, implicó que se tomaran medidas para revertir la situación. Una de ellas ya ha sido mencionada: los cortes programados del servicio en las diferentes localidades. Otra consistió en organizar campañas de toma de conciencia para generar un cambio cultural y lograr que se consumiese menos agua. Un exdirector de ambiente de una de las municipalidades6, con quien tuve una charla, me decía: «Cuando hay campañas de concientización, la gente cuida el agua. Sacás la campaña, y se vuelve a derrochar. Logramos que las personas consuman 200 litros por día, como en los lugares más avanzados en el cuidado del agua».

La crisis hídrica fue primero experiencia y luego significado. Para construir estos significados sobre la crisis, se produjo un conocimiento sobre la escasez que involucró una movilización social de formas y acciones tendientes a paliar la situación, sea a través de la regulación del consumo, sea mediante el planteamiento de un cambio en los comportamientos «culturales» con relación al recurso. Pero a medida que la crisis se intensificaba, y a juicio de diversos actores, estos cambios no eran suficientes. La crisis debía ser encarada de forma más sistemática: había que organizarse, informarse sobre las causas y elaborar planes o acciones para enfrentarla. El problema del conocimiento sobre las causas de la crisis será analizado más adelante. A continuación nos detendremos en las nuevas formas de organización y cooperación que fueron consideradas necesarias en este contexto.

\section{DE CAMPAÑAS ELECTORALES Y PROPUESTAS EN TORNO A LA CRISIS: ¿QUÉ VAN A HACER LOS POLÍTICOS Y QUÉ PUEDEN HACER LOS VECINOS?}

Entre junio y agosto del año 2011, y en gran parte de la provincia de Córdoba, transcurrió un período de campaña electoral con motivo del desarrollo de las elecciones a intendentes, legisladores y gobernador. Lo que aquí nos interesa de esta campaña es que fue un tiempo en el cual los debates en torno a la crisis hídrica

6 Las dependencias del Poder Ejecutivo Municipal están organizadas en secretarías y direcciones. Las direcciones tienen un estatus burocrático-administrativo de menor jerarquía que las secretarías. 
en Sierras Chicas se intensificaron, se profundizaron y comenzaron a expandirse hacia otros horizontes de sentido. La crisis se consideraba por entonces como una cuestión estructural y ya formaba parte de la vida cotidiana de los habitantes. En los ámbitos locales, y en este contexto de campaña, ningún candidato a intendente o legislador pudo esquivar el tema. Sus candidatos opositores, las cooperativas de servicios públicos y la «ciudadanía» en general los interpelaban para que se pronunciaran al respecto en forma contundente y para que dieran a conocer sus propuestas e iniciativas en el caso de que fueran electos. Así, por ejemplo, una de las cooperativas de servicios públicos propuso que todos los candidatos a intendente de una localidad respondieran por escrito un cuestionario sobre el tema. Las preguntas, entre otras, fueron las siguientes:

- ¿Qué medidas concretas propone adoptar su partido en caso de ganar las elecciones para enfrentar los problemas hídricos que afectan a todas las localidades de Sierras Chicas y a Río Ceballos en particular?

- ¿Qué modelo de desarrollo urbano proponen, considerando las condiciones ambientales y el desmesurado crecimiento urbano?

- ¿Qué política ambiental tiene pensado implementar en la reserva hídrica La Quebrada?

De este modo, desde las instituciones de la sociedad civil (en este caso una cooperativa de servicios públicos), las preguntas directas a los políticos fueron una de las maneras de interpelar a los futuros representantes, por entonces en campaña, para que se pronunciaran en torno a la crisis hídrica y la manera de enfrentarla en los niveles locales. La campaña movilizó activamente a una gran diversidad de protagonistas implicados en forma directa en las cuestiones hídricas, propiciando la realización de reuniones entre cooperativas y candidatos a legisladores, de entrevistas periodísticas a científicos e ingenieros, la elaboración de spots de campañas, conferencias sobre los recursos hídricos o eventos políticos, etc., situaciones y eventos que hicieron que la actividad social en torno a la crisis se viera concentrada, intensificada y profundizada.

Así, por ejemplo, y motivados por la intensa actividad social en torno a los recursos hídricos, algunos candidatos a la gobernación de la provincia visitaron la zona de las Sierras Chicas para escuchar a los vecinos, debatir en torno a la crisis, y plantear eventuales propuestas a futuro. Tuve la oportunidad de participar de un evento de campaña realizado en Río Ceballos por uno de los candidatos a gobernador que se postulaba por un partido llamado «Frente Cívico». El evento consistió en una charla centrada en la cuestión hídrica a la cual asistieron entre 
150 y 180 personas de distintas localidades, principalmente miembros de las cooperativas de servicios públicos o agencias municipales relacionadas con el tema del agua. El eje del evento fue la presentación del programa de gestión hídrica para la provincia de Córdoba que el candidato y su equipo técnico habían elaborado para poner en práctica en caso de ganar las elecciones.

El programa planteaba la creación de una estructura ministerial que se encargara exclusivamente de las cuestiones hídricas, un ámbito que hasta entonces estaba bajo la órbita de una Subsecretaría. Es decir, el agua se encontraba en un nivel de gestión inferior al propuesto por este partido. Fue José Manuel de la $\mathrm{Sota}^{7}$, ganador de las elecciones, quien finalmente jerarquizó el problema hídrico al crear el Ministerio de Agua, Ambiente y Energía como una de las primeras medidas de gobierno.

El período de campaña fue, así, un tiempo en el que el debate en torno a la crisis se concentró y en el cual se produjo información, se intercambiaron ideas y se debatió, a tal punto que, en las zonas más afectadas, era casi el único tema que ocupaba a la opinión pública.

Como resultado de esta intensificación de las dinámicas sociales en torno al agua, no solo se crearon nuevas reparticiones del estado provincial sino también nuevas agrupaciones de vecinos, mientras que otras agrupaciones ya existentes vislumbraron un impulso en su accionar. En la localidad de La Granja, por ejemplo, se creó la Mesa de Agua y Ambiente, una agrupación de vecinos que comenzó a reunirse luego de que el intendente de esa localidad convocara a un comité de emergencia para enfrentar la crisis hacia fines del año 2011, ya concluida la campaña electoral. Algunos de los vecinos que participaron de dicha convocatoria acordaron continuar con las reuniones y trabajar a largo plazo, y no solo cuando no hay agua para tomar o bañarse. Sobre aquellas primeras reuniones con el intendente, un miembro de la Mesa me comentaba lo siguiente: la gente fue con mucha bronca, fueron a increpar, fue contestatario y catártico. Mucha gente fue a hacer catarsis.

La idea de continuar con las reuniones - me comentaron - fue la de llevar la cuestión a algo que se transforme de catártico en propositivo, que proponga medidas y proyectos. Esta intención propositiva movilizó, entonces, la búsqueda de información para delinear, en principio, un estado de situación. En primer lugar,

Tres veces gobernador de la provincia de Córdoba por el justicialismo: durante dos períodos consecutivos entre los años 1999 y 2007 , y nuevamente entre 2011 y 2015 . Pertenece a una línea del justicialismo diferente de aquella que gobernó a la Argentina entre 2003 y 2015. 
en cuanto a la red de agua y el sistema de distribución. En una de las primeras reuniones de la Mesa que tuve oportunidad de participar, un ingeniero que es miembro de esta agrupación les contaba a los presentes el funcionamiento de una parte del sistema de distribución de agua de la localidad:

Es una obra de hace cuarenta años, está en perfecto estado [...]. Lo grandioso de esta obra es que va todo por gravedad, no se gasta ni un solo centavo en bombas [...]. Pero parece ser que el caño de salida tiene un diámetro que no aguanta el caudal que sale de los filtros [...]. Es una obra que tiene capacidad para distribuir mucha más agua de lo que distribuye.

$\mathrm{Si}$, como decíamos, la primera información sobre la crisis para los habitantes de las sierras fue la falta de agua en las redes de distribución, es lógico que la situación de las redes, su capacidad y estado, hayan sido uno de los primeros conocimientos que los vecinos agrupados comenzaron a producir. De esta manera, la información es valorada en términos de un conocimiento que oficia como base para las propuestas de acción. Quiero remarcar que esta información fue y es activamente buscada. Un miembro de la Asamblea de Vecinos Autoconvocados de Salsipuedes, la localidad en la que todos aquellos con quienes entré en contacto afirmaban que se vivía la crisis en forma más intensa, me comentaba que «si vas a la municipalidad a pedir el mapa de la red de agua, no lo tienen; no existe. Porque se fue haciendo a los ponchazos».

En este sentido, y en cuanto a la red de distribución, las agrupaciones se enfrentaron también a la falta de información. En el marco de esta búsqueda, durante una de las reuniones de la Asamblea mencionada, se debatió respecto de la presentación o no de un amparo ambiental por supuestas extracciones ilegítimas de agua por emprendimientos inmobiliarios y mineros. Al respecto, la Asamblea había entrado en contacto con un abogado. Esto decía uno de los miembros que había conversado con él:

El abogado tiene el dato de que hay varios emprendimientos inmobiliarios de De La Sota ahí arriba, que están sacando el agua, y un emprendimiento minero de tungsteno que, según tengo entendido, es un mineral liviano que se lava con agua. Bueno, están sacando el agua de ahí y de los ríos de La Quebrada [...]. El abogado, teniendo ese dato, necesita de alguien que se sume a la demanda, y él la haría. Yo le dije que tenemos que sumar el tema de la red de distribución de agua, que está obsoleta.

Es importante aclarar que, para nuestro argumento, es irrelevante si este tipo de información es verdadera o falsa en el sentido de su comprobación fáctica, 
pues, como afirma el antropólogo E. Leach, para los actores que los producen estos relatos son parte de historias que existen y se preservan «con objeto de justificar las actitudes y acciones del presente» (1976, p. 108).

Respecto de otras cuestiones que atañen directamente al tema del conocimiento sobre la crisis, la Asamblea de Vecinos Autoconvocados organizó una charla con un biólogo que vive en la localidad. La charla fue organizada para desmitificar, con información de base científica, ciertas versiones y relatos que circulaban en la localidad respecto de la situación hídrica de la zona. No tuve oportunidad de participar de esa reunión en particular, pero sí de charlar con aquel biólogo luego de realizado el encuentro. Al respecto, me comentaba lo siguiente: « ¡Hay cada mito...! Me llamaron de la Asamblea el otro día para desmitificar ciertas cosas, como por ejemplo que el agua viene de la Cordillera de los Andes, o que no sé dónde hicieron un agujero en la sierra y se la están llevando para no sé qué cosa...».

Así, otro tipo de información que es valorada - y por lo tanto activamente buscada - es la información con base científica. En el próximo apartado entraremos en detalle sobre esta cuestión. Cabe antes insistir en el carácter intencional de la búsqueda de información, pero también en la corrección o el «ajuste» de información considerada como errónea (p.e., los mitos a los cuales se refiere el biólogo). Particularmente, quisiera hacer notar que los procesos que aquí llamo como de producción de conocimiento requirieron las condiciones de posibilidad para su realización. Estas condiciones tuvieron que ser creadas: fue en el marco de una asamblea que la posibilidad de convocar a un biólogo para que brindase información precisa se hizo efectiva, y fue gracias a esta forma de agrupamiento como la búsqueda de información se imbrica y forma parte de la creación de «nuevas» formas de cooperación social; es decir, de nuevas relaciones sociales ${ }^{8}$.

Pasemos ahora al tratamiento en profundidad de las formas de conocimiento que implican la búsqueda de información científica. La dinámica es la misma: cambia solo el tipo de información. Como adelantamos, para los actores la ciencia puede aportar información respecto de las causas de la crisis, información que debe ser compartida y difundida entre la mayor cantidad de gente posible. Esto solo fue realizable, para los protagonistas, a través de la organización y la coordinación mutua de las actividades y acciones concretas.

8 Por supuesto que la «novedad» no reside en la forma de asamblea, sino en el hecho de que se constituya una asamblea para tratar los problemas hídricos en el marco de estas iniciativas de intervención sobre la crisis hídrica, y que a partir de estas formas de cooperación se produzca conocimiento como parte constitutiva de la creación de esas mismas relaciones. 


\section{LA CIENCIA ENTRA EN ESCENA: CONOCER LA VEGETACIÓN NATIVA, LA CUENCA Y EL AMBIENTE}

En la zona de Sierras Chicas, diversos «especialistas» trabajan directamente involucrados con la política de los recursos hídricos, como biólogos que asesoran a las cooperativas de servicios públicos, funcionarios de las áreas de medio ambiente de las municipalidades, o incluso profesionales que participan, en calidad de vecinos, en las diferentes agrupaciones. Estos especialistas eran reiteradamente interpelados por los periodistas locales para que explicaran las causas de la crisis hídrica. Los factores climáticos y el aumento del consumo, decían, eran parte de las explicaciones, pero estas no se agotaban allí. Así me lo explicó aquel exdirector de ambiente antes citado:

A lo que apuntamos es que el agua sea retenida en la tierra, y esto solo se puede hacer si tenés vegetación. Bosque, monte, pastizal, y que escurra en forma lenta para formar vertientes, y que sean las vertientes las que llenen el dique [...]. El sobrepastoreo es un problema grave [para la preservación del pastizal]. Los incendios son otro gran problema.

La solución a estos problemas es, entonces, mantener sanas las cuencas. Es decir, mantener y preservar la vegetación, sobre todo la autóctona. Quienes proponían esto como la clave del manejo de la crisis son científicos de disciplinas como la biología o la geografía, ingenieros agrónomos y civiles, o arquitectos. Algunos de estos profesionales universitarios se radicaron en la zona buscando ambientes más amigables para vivir que la ciudad. Participan activamente en los debates públicos y en las iniciativas de acción en torno a la crisis hídrica, y no es extraño, entonces, que la información que ellos brindan sea socialmente valorada por una serie de agrupaciones informales que comenzaron a surgir al mismo tiempo que la crisis se hacía cada vez más profunda. Muchos de estos especialistas participan de estas nuevas agrupaciones, y también han tenido o tienen cargos de gestión en algunas de las agencias de gobierno locales. A lo largo del tiempo, y por influencia de estos profesionales, el problema del agua, y del ambiente en general, empezó a significarse en términos del cuidado de las cuencas?.

$9 \quad$ La problemática hídrica en términos de cuenca comenzó a ser difundida en el mundo luego de una de las primeras experiencias en Francia, que en 1964 comienza un proceso de modernización de su gestión hídrica en el país. A más de cuarenta años de su creación, «la actual estructura de gobernanza francesa de las aguas ocupa un lugar destacado en el debate internacional sobre modelos de gestión de recursos naturales» (Martins, 2008). De hecho, fue la 
Las instancias y los medios de circulación de la información de origen científico son muy diversos. Por razones de espacio, me concentraré solo en algunas situaciones: aquellas que por su magnitud, cualidad, nivel de organización y sistematicidad, se destacan por sobre otras.

Así, por ejemplo, la Municipalidad de La Granja organiza anualmente, desde hace ya seis años, una Feria del Libro que publicita como el evento más convocante de las Sierras Chicas y que se realiza durante los días feriados de la Semana Santa. La Mesa de Agua y Ambiente logró que la Municipalidad autorizara, para cada edición de la Feria, el armado de un espacio en donde presentar sus actividades, propuestas y objetivos a quienes se acerquen, y ofrecer charlas de diferentes especialistas que giran alrededor de temas ambientales y de los recursos hídricos. Para cada edición de la Feria del Libro, toda la programación de las actividades de la Mesa de Agua para el público implica la puesta en escena de diferentes tipos de conocimiento.

Tuve oportunidad de participar en la logística y organización de las actividades planificadas durante la Feria de 2014. Allí, la primera actividad consistió en la exposición de una arquitecta que había participado en la elaboración del último plan de ordenamiento urbano de la localidad, y de un ingeniero agrónomo que trabaja en temas de geografía en la universidad y que se había desempeñado asimismo como funcionario en el área ambiental en otra de las localidades de las sierras (que no entran en nuestra área de estudio). Ambos hablaron de sus respectivas especialidades y actividades, focalizando en el ordenamiento territorial como base de la planificación urbana y ambiental. El ingeniero, por su parte, y a pedido de la Mesa de Agua y Ambiente que organizaba la actividad, trajo mapas e imágenes satelitales de las cuencas de las Sierras Chicas, explicando la geografía hídrica de la zona. Luego expusieron dos miembros del Concejo Deliberante de la localidad de Río Ceballos, quienes explicaron su propuesta de poner en práctica políticas de construcción de viviendas ambientalmente amigables, basadas en métodos de construcción que generen un impacto mínimo en la vegetación nativa, una de las grandes preocupaciones de todas las agrupaciones de las Sierras Chicas con relación al problema ambiental e hídrico.

De esta manera, se puso en juego una serie de conocimientos que los actores consideran como necesarios en el marco de su intervención sobre los problemas socioambientales. Este conocimiento de base científica aporta un elemento común

base para lo que actualmente se conoce como gestión integrada de los recursos hídricos. Para más detalles, véanse Gleick (2000) y Martins (2008). 
a la gran diversidad de situaciones que se vive en cada localidad. Por ejemplo: si para preservar el recurso hídrico se deben mantener sanas las cuencas, tal como adelantamos que los actores consideran, uno de los elementos centrales de esta salud es el mantenimiento y preservación de la vegetación nativa. Entonces, no es casualidad que el conocimiento de la vegetación nativa sea altamente valorado ${ }^{10}$. Con la intención de conocer y dar a conocer la vegetación nativa, se brindó también una charla sobre las distintas variedades de plantas, árboles y flores autóctonas, que también abarcó sus potenciales usos decorativos ${ }^{11}$, alimenticios y medicinales. Quienes defienden la preservación de la vegetación nativa consideran que el conocimiento de las funciones biológicas que cumple, de sus nombres, sus variedades y sus propiedades, aportará a la creación de conciencia por el cuidado del bosque y del monte de las sierras, aspecto central para mantener la salud de las cuencas.

Pero no es solo durante la Feria del Libro que esta agrupación se preocupa por difundir información respecto del ambiente y del agua. Durante un proceso que llevó a la declaración de reserva natural a unos terrenos de la Fuerza Aérea en el norte de las Sierras Chicas, la Mesa armó un volante difundiendo el tema, que llevaba por título «Sembremos el agua». Me comentaba al respecto un miembro de esta agrupación que:

La mesa también se dedicó a generar charlas, conferencias, para generar una conciencia dentro de la comunidad de lo que estábamos haciendo [...]. Como dentro de la reserva hay el nacimiento de algunos ríos que forman una de las cuencas que nosotros tenemos acá $[\ldots]$ y también está el nacimiento de los ríos que forman la cuenca del Río Carnero, entonces se hizo una reunión, un taller, y se logró un tratado de la cuenca del Río Carnero, o sea todas las poblaciones que estaban involucradas que dependían de esa cuenca, ¿no es cierto?

Una nota de prensa afirmaba al respecto que:

El tratado firmado - al que se agregarán las firmas de los intendentes y jefes comunales de Jesús María, Agua de Oro, La Granja, Ascochinga, Colonia

10 Un dibujo de un árbol nativo es, justamente, el logotipo identificador de la agrupación que organizó esta actividad. La importancia de la vegetación autóctona reside en su menor consumo de agua, en contraste con la foránea, y en el efecto esponja que produce al retenerla en la superficie mientras la va dosificando gradualmente para que escurra lentamente hacia la tierra. La vegetación foránea sería ineficaz en este efecto.

11 La ponderación del valor estético de la vegetación nativa implica la intención de generar conciencia en virtud de la «parquización» de jardines y espacios privados y públicos, para así evitar que se utilice vegetación foránea. 
Tirolesa, Estación General Paz, La Cumbre, La Pampa, Cerro Azul y El Manzano- compromete a las autoridades a trabajar por el futuro de la cuenca y el recurso del agua ${ }^{12}$.

En este sentido, el conocimiento forma parte de la creación de relaciones sociales, al dar cuenta de una unidad: la cuenca como unidad espacial compartida.

En el año 2012, se crea la Coordinadora Ambiental y de Derechos Humanos Sierras Chicas, que surgió ante el reconocimiento de que los problemas comunes con los que lidiaban las agrupaciones de las diferentes localidades eran problemas regionales. Los objetivos de la Coordinadora son, entre otros, luchar por la vida, el agua y la naturaleza en las Sierras Chicas, apoyando las problemáticas ambientales nacionales y priorizando el debate y el bien común de las localidades cercanas. Con la puesta en funcionamiento de la Coordinadora, los diversos conflictos que hasta entonces habían tenido un carácter específicamente local comenzaron a ser impulsados y visibilizados como enmarcados en una problemática común a todas las sierras (texto del autor, 2015).

Una de las primeras iniciativas para visibilizar a la organización y sus actividades fue la realización de una cruzada de las Sierras Chicas, un recorrido por las diversas localidades con el propósito de ir hilando todo lo que está pasando en cada una de las localidades en términos ambientales. La Coordinadora nuclea aproximadamente quince agrupaciones de prácticamente todas las localidades de las Sierras Chicas, la mayoría de las cuales tiene un trabajo previo con las problemáticas socioambientales locales y específicas de cada lugar. Sin embargo, su creación puede entenderse como resultado y condición de un nuevo impulso a las actividades y a la visibilidad pública del problema ambiental. En un cuadernillo de esta agrupación, se afirma lo siguiente:

Los grandes medios reducen las causas del problema a la falta de lluvias y a la sequía. Sin embargo, la crisis hídrica de las Sierras Chicas se presenta como un conflicto socioambiental, generado por la combinación entre las condiciones ambientales de la zona por un lado, con el clima, el suelo y las cuencas.

En el marco de las problemáticas ambientales, la información científica y técnica provee narrativas cohesivas (Bixler, 2013). La narrativa sobre la salud de las cuencas se vuelve así un marco de sentido para la acción. Pero, al mismo tiempo, el objeto de la acción - la cuenca en sí misma - motiva la creación de nuevas relaciones sociales, como la Coordinadora o como el tratado antes

12 http://www.prensasierraschicas.com.ar/?p=8782 
mencionado. En este proceso, la crisis hídrica empezó considerarse como parte de una crisis socioambiental más amplia. En junio de 2013 se creó en La Granja, por ejemplo, por primera vez, una Dirección de Ambiente municipal. Si bien ya se venía discutiendo la posibilidad, esta creación se funda en un largo proceso de circulación de conocimiento que sienta las bases de una necesidad social de intervenir sobre la crisis.

Habíamos afirmado, siguiendo a Bixler (2013), que la manera en que la información científica era usada y apropiada operaba produciendo narrativas cohesivas. Pues bien, debemos dar ahora un paso más, ya que la cohesión que expresa esta narrativa solo puede entenderse atendiendo, al mismo tiempo, al lugar que ella ocupa en los procesos conflictivos.

Me voy a detener brevemente en una situación que ocurrió en el contexto de una manifestación en contra de un emprendimiento inmobiliario que se instaló en las cercanías de la captación de agua de una cooperativa de servicios públicos. Los vecinos agrupados — que son también socios de la cooperativa - impulsan desde hace un tiempo medidas políticas y judiciales para evitar que se construyan viviendas en el lugar, ante un posible impacto ambiental negativo de las construcciones y posterior poblamiento de la zona en la calidad del agua.

En la entrada al emprendimiento, me mostraron las razones de su malestar e irritación respecto del discurso ecológico de la empresa, que afirmaba que «parquizaría» la zona con árboles nativos. Efectivamente están parquizando con árboles nativos, me dijeron. Mirá, todos esos son aguaribay, recién plantaditos. Sí, son árboles nativos, pero son nativos de otro lado. Como acto de protesta frente a la presencia de árboles no autóctonos plantados por la empresa, una de las acciones que se llevaron adelante fue el bombardeo del lugar con bombas de semillas. Estas bombas, de forma redonda y de dimensiones que oscilan entre el tamaño de una pelota de tenis de mesa y una pelota de tenis de cancha, están compuestas de arcilla, tierra y semillas de flora autóctona. El objetivo es lanzarlas a mano en zonas a las cuales no se puede sembrar de otra manera, como pueden ser los terrenos privados en donde está prohibido el acceso, lugares muy escarpados, o cuya accesibilidad está restringida por cualquier otra razón ${ }^{13}$. Cada uno de los presentes debía tomar una de estas bombas y arrojarla donde creyera conveniente, siguiendo solo un par de indicaciones como para no desperdiciar ninguna de las

13 En este caso, se trató de evitar el ingreso al espacio del emprendimiento inmobiliario para evitar cualquier tipo de reclamo legal por la empresa que pudiera impactar negativamente en el desarrollo del conflicto, pues se encuentra actualmente en instancias judiciales. 
«municiones». Luego de unas palabras del presidente de la cooperativa de agua, la zona fue bombardeada.

Así, la narrativa sobre las cuencas y la vegetación nativa unifica a todas las agrupaciones en una suerte de causa común: la preservación del bosque, que se vuelve al mismo tiempo una arena de conflicto con los emprendimientos inmobiliarios y las actividades de construcción en general (junto con todo tipo de actividad antrópica) que incidirían negativamente en la salud de las cuencas.

\section{PALABRAS FINALES}

Hemos realizado un recorrido que tomó como punto de partida el momento en que la crisis hídrica pasó de manifestarse como experiencia concreta y sistemática, en términos de falta de agua, a un problema socioambiental a resolver. El primer paso de los actores protagonistas de la política de los recursos hídricos en este sentido, tal como lo hemos descrito, fue comenzar a buscar y hacer circular información que se volvió un conocimiento sobre la escasez, y que llevó asimismo a buscar información respecto de sus causas. Esta búsqueda activa generó la necesidad de organización y coordinación de las actividades, por lo cual se fueron creando, en virtud de esta necesidad, nuevas relaciones sociales. Algunas de ellas, aquellas en que las agencias estatales estaban involucradas en forma más directa, comenzaron a tomar forma institucional, modificando la estructura burocrática del Estado. Otras, sin embargo, surgieron con un carácter menos formal, como las asambleas de vecinos que se conformaron para intervenir sobre los problemas generados por la crisis. Hemos analizado este proceso de producción social en términos del lugar que en él ocupó la información factual, tanto científica como no científica, devenida, por razón de su lugar en esta productividad social, en conocimiento.

Como hemos mostrado, en el contexto de la política de los recursos hídricos en Sierras Chicas, el conocimiento juega un triple papel: en primer lugar, sienta las bases de una cohesión e implica la puesta en escena de un estado de situación común a todas las localidades de las Sierras Chicas. Así, el conocimiento unifica a las distintas agrupaciones de las diferentes localidades en una suerte de causa común: la preservación del bosque nativo para mantener sanas las cuencas. En este sentido, la circulación de conocimiento genera tanto la necesidad de cooperación y coordinación como una diversidad de instancias de intercambio y relación entre las agrupaciones. Pero también, en segundo lugar, genera instancias de intercambio «hacia afuera», es decir, con los vecinos que no participan 
activamente de estas iniciativas, o con las personas que visitan las localidades serranas durante los fines de semana, que se transforman así en una audiencia que otorga reconocimiento y visibilidad social a las actividades de las agrupaciones. La visibilidad es clave, pues estos grupos, como la Mesa de Agua y Ambiente de La Granja, la Coordinadora, o la Asamblea de Vecinos Autoconvocados de Salsipuedes, tienen la intención de incidir en forma activa y efectiva en el curso de los acontecimientos relativos al manejo de la crisis. En tercer lugar, el conocimiento genera las condiciones para que el objeto de ese conocimiento (la cuenca, la vegetación nativa) opere como una arena de conflicto entre diversos actores.

Líneas atrás decíamos, siguiendo a Godelier (1989), que la creación de nuevas relaciones sociales solo es posible si estas son pensables y consideradas como necesarias. A lo largo del trabajo propusimos centrarnos en ambas cuestiones. Así, el conocimiento podría entenderse tanto como la «sustancia generadora» como la «fuerza productiva» de la acción e intervención sobre la crisis, que impulsaron la creación de nuevas relaciones sociales. En este sentido, el artículo también se inserta en la problemática más general del análisis del cambio social.

Un interrogante que queda abierto para futuras indagaciones nos lleva plantear por qué el conocimiento sobre la naturaleza en términos de las cuencas hídricas es socialmente valorado por sobre otro tipo de conocimiento. Nuestra hipótesis de trabajo es, al respecto, que si bien este conocimiento oficia como sustancia generadora de las relaciones sociales en los niveles locales, es producto de una narrativa internacional que oficia de «fuerza legitimante» de las intervenciones sobre los recursos hídricos y el ambiente. El desafío analítico será, entonces, desentrañar las relaciones o mediaciones entre la especificidad de lo local y los procesos que se dan a escala internacional.

\section{REFERENCIAS BIBLIOGRÁFICAS}

Balbi, Fernando (2006). Entre el futuro del recurso y el futuro de los hijos. Usos de expresiones y términos ambientalistas entre los pescadores del delta del Río Paraná. Cuadernos de Antropología Social, 26, 87-105.

Barth, Frederik (1995). Cosmologies in the making. A generative approach to cultural variation in inner New Guinea. Cambridge: Cambridge University Press.

Barth, Frederik (2000). O guru, o iniciador e outras variações antropológicas. En O guru eo iniciador: transaçŏes de conhecimento y modalgem da cultura no sudeste de Ásia e na Melanésia (pp. 141-166). Rio de Janeiro: Contracapa. 
Bixler, R. Patrick (2013). The political ecology of local environmental narratives: power, knowledge and mountain caribou conservation. Journal of Political Ecology, 20, 273-285.

Blois, María Paula (2016). Ciencia y glifosato: interpretando órdenes. Una investigación en la prensa en el contexto argentino. Cuadernos de Antropología Social, $43,73-79$.

Carabajal, María Inés (2016). Servicios climáticos y producción de conocimiento científico útil. Estudio de caso en una comunidad climática de Argentina. Cuadernos de Antropología Social, 43, 33-49.

Descola, Philippe (2012). Más allá de la naturaleza y la cultura. Buenos Aires: Amorrortu.

Escobar, Arturo (1999). After Nature. Steps to an antiessentialist political ecology. Current Anthropology, 40(1), 1-30. https://doi.org/10.1086/515799

Ferrero, Brian (2006). «La ecología» de los colonos. Búsquedas de inclusión en un territorio ambientalista. Anuario de Estudios en Antropología Social 2005. CAS-IDES, 187-197.

Gleick, Peter (2000). The changing water paradigm. A look at twenty-first century water resources development. Water International, 25(1), 127-138. https://doi. org/10.1080/02508060008686804

Godelier, Maurice (1989). Lo ideal y lo material. Pensamiento, economía, sociedades. Madrid: Taurus.

Hidalgo, Cecilia y Adriana Stagnaro (2016). Antropología de la ciencia y la tecnología. Cuadernos de Antropología Social, 43, 9-11.

Hukkinen, Janne (2014). Model of the social-ecological system depends on model of the mind: contrasting information-processing and embodied views of cognition. Ecological Economics, 99, 100-109. https://doi.org/10.1016/j.ecolecon.2014.01.017

Ingold, Tim (2000). The perception of Environment: Essays on Livelihood, Dwelling and Skill. Londres: Routledge. https://doi.org/10.4324/9780203466025

Koberwein, Adrián (2015). La producción de relaciones sociales en el marco de la política de los recursos hídricos en Sierras Chicas, Córdoba - Argentina. De la coordinación de las acciones a la región como valor. Argumentos. Revista de crítica social (17), 68-93.

Koberwein, Adrián (2015b). Espacio, territorio y la política del agua en las Sierras Chicas de Córdoba - Argentina. Sociedade \& Natureza, 27(2), 239-254.

Leach, Edmund (1976). Sistemas politicos de Alta Birmania. Estudio sobre la estructura social Kachin. Barcelona: Anagrama. 
Leff, Enrique (2000). Espacio, lugar y tiempo: la reapropiación social de la naturaleza y la construcción local de la racionalidad ambiental. Desenvolvimiento e Meio Ambiente (1), 57-69. https://doi.org/10.5380/dma.v1i0.3057

Marcus, George (2000). Etnografía en/del sistema mundo. El surgimiento de la etnografía multilocal. Alteridades, 11(22), 111-127.

Martins, Rodrigo Constante (2008). Sociologia da governança francesa das águas. RBSC (23)67, 83-101. https://doi.org/10.1590/s0102-69092008000200007

Marx, C. (2000)[1867]. El capital. Crítica a la economía política. Buenos Aires: Siglo Veintiuno.

Marx, C. y F. Engels (2005)[1845]. La ideología alemana. Buenos Aires: Santiago Rueda Editora.

Leite Lopes, José Sergio (2006). Sobre processos de «ambientalização» dos conflictos e sobre dilemas da participação. Horizontes Antropológicos, 12(25), 31-64. https:// doi.org/10.1590/S0104-71832006000100003

Pinto Ribeiro, Fernando (2012). O Paradigma ambiental na globalização neoliberal: da condição crítica ao protagonismo de mercado. Sociedade \& Natureza, 24(2), 211-226.

Stagnaro, Adriana (2015). Representaciones culturales e identitarias en cambio: habitus científico y políticas públicas en ciencia y tecnología en la Argentina. Cuadernos del Centro de Estudios en Diseño y Comunicación, 52, 313-326.

Swartz, M., V. Turner y Arthur Tuden (1994). Antropología política: una introducción. Alteridades, 1(8), 101-126.

Turner, Terence. (2008). Marxian value theory. An anthropological perspective. Anthropological Theory, 8(1), 43-56. https://doi.org/10.1177/1463499607087494

Vázquez, Inés (2017). Tecnópolis: ciencia y contexto en exposición. Estudios en Antropología Social-Nueva Serie, 1(2), 58-72. 